\title{
Characterization and optimization of a high-efficiency AIGaAs-On-Insulator-based wavelength converter for 64- and 256-QAM signals
}

Da Ros, Francesco; Yankov, Metodi Plamenov; Porto da Silva, Edson; Pu, Minhao; Ottaviano, Luisa; Hu, Hao; Semenova, Elizaveta; Forchhammer, Søren; Zibar, Darko; Galili, Michael

Total number of authors:

12

Published in:

Journal of Lightwave Technology

Link to article, DOI:

10.1109/JLT.2017.2722013

Publication date:

2017

Document Version

Peer reviewed version

Link back to DTU Orbit

Citation (APA):

Da Ros, F., Yankov, M. P., Porto da Silva, E., Pu, M., Ottaviano, L., Hu, H., Semenova, E., Forchhammer, S., Zibar, D., Galili, M., Yvind, K., \& Oxenløwe, L. K. (2017). Characterization and optimization of a high-efficiency AIGaAs-On-Insulator-based wavelength converter for 64- and 256-QAM signals. Journal of Lightwave Technology, 35(17), 3750-3757. https://doi.org/10.1109/JLT.2017.2722013

\section{General rights}

Copyright and moral rights for the publications made accessible in the public portal are retained by the authors and/or other copyright owners and it is a condition of accessing publications that users recognise and abide by the legal requirements associated with these rights.

- Users may download and print one copy of any publication from the public portal for the purpose of private study or research.

- You may not further distribute the material or use it for any profit-making activity or commercial gain

- You may freely distribute the URL identifying the publication in the public portal 


\title{
Characterization and optimization of a high-efficiency AlGaAs-On-Insulator-based wavelength converter for 64- and 256-QAM signals
}

\author{
Francesco Da Ros, Member, IEEE, OSA Metodi P. Yankov, Member, IEEE, \\ Edson P. da Silva, Student Member, IEEE, OSA Minhao Pu, Luisa Ottaviano, Hao Hu, Member, OSA, \\ Elizaveta Semenova, Søren Forchhammer, Member, IEEE Darko Zibar, Member, IEEE \\ Michael Galili, Member, IEEE Kresten Yvind, and Leif K. Oxenløwe, Member, OSA
}

\begin{abstract}
In this paper, we demonstrate wavelength conversion of advanced modulation formats such as 10-GBd 64-QAM and 256-QAM with high conversion efficiency over a 29-nm spectral window by using four-wave mixing in an AlGaAs-On-Insulator (AlGaAsOI) nano-waveguide. A thorough characterization of the wavelength converter is reported, including the optimization of the AIGaAsOI nano-waveguide in terms of conversion efficiency and associated bandwidth and the analysis of the impact of the converter pump quality and power as well as the signal input power. The optimized converter enables generating idlers with optical signal-to-noise ratio (OSNR) above $30 \mathrm{~dB}$ over a 29-nm bandwidth leading to error-free conversion of 64-QAM and 256-QAM with OSNR penalty below $1.0 \mathrm{~dB}$ and $2.0 \mathrm{~dB}$ respectively. The generated idlers exhibit an OSNR margin to the chosen forward error correction thresholds of $>3 \mathrm{~dB}$ and $>7 \mathrm{~dB}$ for 64-QAM and 256-QAM, respectively, that can be used for transmission after conversion.
\end{abstract}

Index Terms-four-wave mixing, integrated waveguides, quadrature amplitude modulation, coherent communications.

\section{INTRODUCTION}

C URRENT wavelength division multiplexed (WDM) communication systems rely on wavelength channels to route data through the network. In order to improve the wavelength utilization, as well as to decrease the blocking probability, it is not only desirable but strongly beneficial to shift a data channel from one wavelength to another [1]. The ability to provide such operation without converting the data back to the electrical domain, i.e. performing all-optical wavelength conversion, enables processing multiple channels simultaneously, as well as reducing the number of required converters [1]. Additionally, all-optical wavelength conversion based on four-wave mixing (FWM) is a fundamental building block to provide more advanced functionalities such as low noise amplification [2], phase-sensitive amplification and regeneration $[3]-[5]$, and Kerr nonlinearity mitigation through optical phase conjugation [6]-[9].

Advanced modulation formats, such as high-order quadrature amplitude modulation (QAM), are being deployed in optical networks to enable higher spectral efficiency transmissions improving the overall transmission rate. In order for all-optical wavelength converters to be considered for

Department of Photonics Engineering, Technical University of Denmark, 2800 Kgs. Lyngby, Denmark: e-mail: fdro@fotonik.dtu.dk practical applications, they must be able to accommodate the increasingly stringent requirements in terms of phase noise tolerance and optical signal-to-noise ratio (OSNR) degradation set forth by QAM signals. Wavelength converters should therefore provide low-penalty and modulation-format independent operation. Low penalty wavelength conversion for signals up to 64-QAM has been demonstrated using FWM in highly nonlinear fibers [10], [11]. Although efficient and mature, fiber-based converters are ultimately limited by the modest nonlinearity of highly nonlinear fibers (HNLFs) and by stimulated Brillouin scattering. The former leading to long fiber lengths and the latter requiring mitigation techniques introducing additional complexity in the system [7], [8].

Integrated solutions based on nonlinear materials such as silicon-based compounds have been the target of a strong research focus due to their enhanced Kerr nonlinearity which enable more compact devices. Alternative materials such as chalcogenide [12] and periodically poled lithium niobate [13], [14] waveguides have also shown promising results. However, silicon-based compounds may be more attractive as they could enable benefiting from the well established CMOS fabrication technology. In this regard, several material platforms have been investigated, spanning from crystalline [9] and amorphous silicon [15], to silicon-germanium [15], [16] and siliconnitride [17], as well as high index doped glass [18].

Nonlinear processing in crystalline silicon is mainly hindered by two photon absorption (TPA), and consequently free-carrier effects at telecom wavelengths. While successful approaches have been reported to actively mitigate the impact of free-carrier effects [4], materials such as amorphous silicon and silicon-germanium allow to decrease the impact of TPA as reported in [15], [19]. Successful demonstration of wavelength conversion for QAM signals have been recently reported in both platforms [15], [20] without requiring active TPA suppression. However, these materials are still affected by significant propagation loss $(\approx 5 \mathrm{~dB} / \mathrm{cm})$ and further limited by the lower but still present TPA.

Silicon nitride and high-index doped glass alloys can effectively shift the material bandgap such that no TPA is present at telecom wavelength. The bandgap shift comes at the expense of lower Kerr nonlinearity which leads to a low conversion efficiency (CE) and thus only a modest output idler OSNR [18]. 


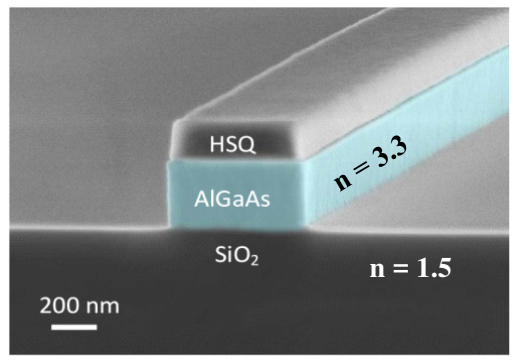

Fig. 1. Colored SEM image after dry etching highlighting the refractive index contrast between the AlGaAs waveguide and the SOI substrate.

A material platform offering high nonlinearity, low propagation loss and a bandgap ensuring TPA-free operation at telecom wavelength is highly desired. AlGaAs has been shown to provide large intrinsic nonlinearity $\left(\mathrm{n}_{2} \approx 10^{-17} \mathrm{~W} / \mathrm{m}^{2}\right)$, and no TPA around $1550 \mathrm{~nm}$ [21]-[24]. To further enhance the effective nonlinearity, we have previously demonstrated the AlGaAs-on-insulator (AlGaAsOI) platform, where moderately low propagation loss $(\leq 1.5 \mathrm{~dB} / \mathrm{cm})$ high-index contrast nanowaveguides can be realized with a high nonlinear coefficient. This platform has enabled efficient nonlinear processes such as FWM for wavelength conversion [25]-[28], and phasesensitive processing [29], as well as self-phase modulation (SPM) based supercontinuum generation [30].

In this work, we extend our previous system investigation [28], by demonstrating error-free wavelength conversion for 64-QAM and 256-QAM signals over most of the C-band (29-nm bandwidth) in an optimized wavelength converter based on a 9-mm long AlGaAsOI nano-waveguide. The waveguide design has been optimized in terms of the conversion efficiency (CE) and conversion bandwidth by tuning the waveguide cross-section and length, as well as the pump power. From a system perspective, signal degradations taking place in the converter are minimized by investigating the impact of the pump laser linewidth as well as the trade-off between output idler OSNR and nonlinear distortion. The high CE provided by $\mathrm{AlGaAsOI}$ is a key enabler for achieving idler OSNR levels in excess of $30 \mathrm{~dB}$ and thus meeting the strict requirements for demodulating error-free 64-QAM and 256-QAM idlers with limited OSNR penalty $(<1 \mathrm{~dB}$ and $<2.0 \mathrm{~dB}$, respectively). Additionally, a significant OSNR margin that can be used for further transmission after the wavelength conversion is reported $(>3 \mathrm{~dB}$ and $>7 \mathrm{~dB}$ for 64-QAM and 256-QAM, respectively).

\section{AlGaAsOI FABricAtion}

The AlGaAsOI nano waveguide has been fabricated from a GaAs/AlGaAs wafer prepared by epitaxial growth, wafer bonding and substrate removal [26], [31]. By tuning the concentration of $\mathrm{Al}$ and $\mathrm{Ga}$, the bandgap of the material can be tuned such that no TPA is present at $1550 \mathrm{~nm}$. The waveguides considered in this work have an $\mathrm{Al}$ percentage of $21 \%$, leading to a bandgap of approx. $1.69 \mathrm{eV}$. The waveguides have been defined by electron-beam lithography

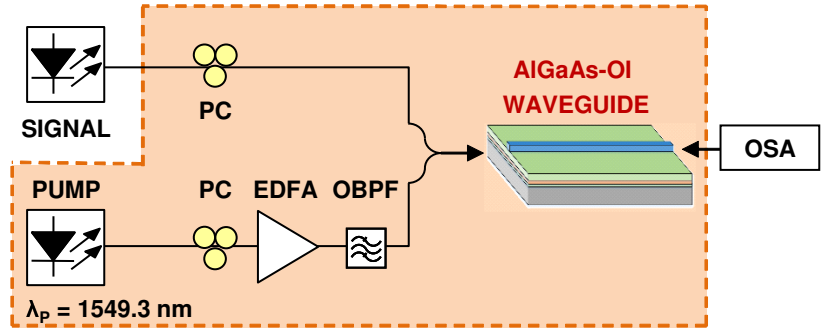

Fig. 2. Experimental setup for nonlinear characterization of AlGaAsOI-based wavelength converter (orange shaded area).

and dry etching using hydrogen silsesquioxane (HSQ) as a hard mask. The measured propagation loss is $1.5 \mathrm{~dB} / \mathrm{cm}$ for the TE mode. In order to increase the coupling efficiency, inverse tapers are implemented leading to a typical coupling loss of $1.4 \mathrm{~dB} /$ facet [32].

A scanning electron microscope (SEM) picture of the waveguide prior to over-cladding deposition is shown in Fig. 1] The smooth sidewalls shown in the picture are critical for achieving low propagation loss and thus high-efficiency FWM.

\section{DISPERSION ENGINEERING}

The ability to tailor the dispersion properties of the wavelength converter by optimizing the waveguide cross-section is a major benefit of integrated devices. Different waveguide dimensions have been investigated, aiming at maximizing the achievable conversion efficiency (CE), as well as the conversion bandwidth.

Several 3-mm long straight waveguides have been fabricated with a height of $290 \mathrm{~nm}$ and a width spanning between $580 \mathrm{~nm}$ and $730 \mathrm{~nm}$. The dispersion curves for these waveguide dimensions have been numerically estimated starting from the material dispersion derived according to [33] and considering a 30-nm width reduction common in our lithography and etching processes. For consistency, all the waveguide dimensions stated in the following refer to the design dimensions. The nonlinear properties of such waveguides have then been investigated by measuring the conversion bandwidth with the experimental setup shown in Fig. 2

The wavelength converter is based on a single-pump FWM stage where the input continuous wave $(\mathrm{CW})$ signal is coupled together with a strong CW pump at $1549.5 \mathrm{~nm}$. The pump has been placed in the middle of the $\mathrm{C}$-band aiming at providing wavelength-conversion operation over the full $\mathrm{C}$-band. The pump has been amplified in an erbium-doped fiber amplifier (EDFA) followed by an optical band-pass filter (OBPF) to remove out-of-band amplified spontaneous emission (ASE) noise. Polarization controllers (PCs) are used to align the state of polarization of the two waves to the TE mode of the AlGaAsOI waveguide where they are injected using a lensed fiber with a spot size of $2.5 \mu \mathrm{m}$. At the output of the waveguide, light is collected through a second lensed fiber and an optical spectrum analyzer (OSA) is used to record the output spectra. The pump power at the input of the waveguide (lensed fiber output) has been set at $18 \mathrm{dBm}$ for this investigation. 


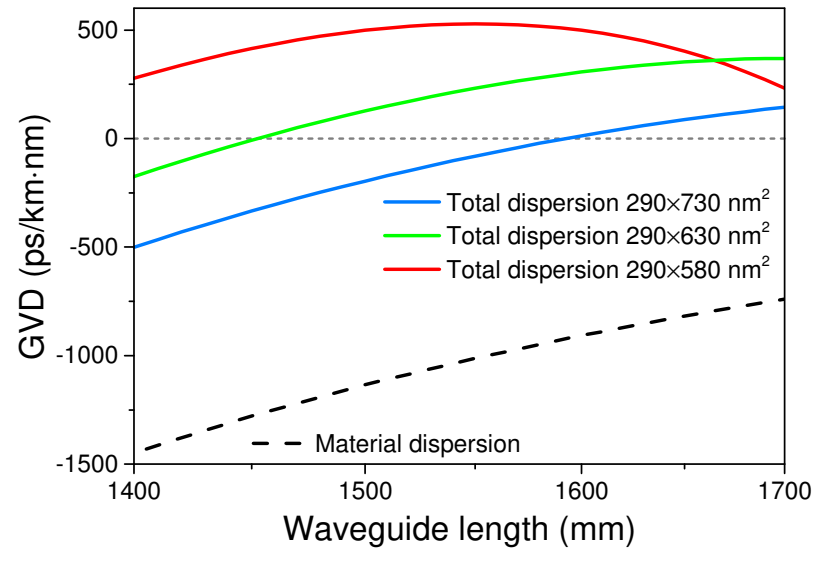

Fig. 3. Numerically calculated material (dashed) and total (solid) dispersion for fabricated waveguide with design cross-sections of $290 \times 580 \mathrm{~nm}^{2}$, $290 \times 630 \mathrm{~nm}^{2}$ and $290 \times 730 \mathrm{~nm}^{2}$.

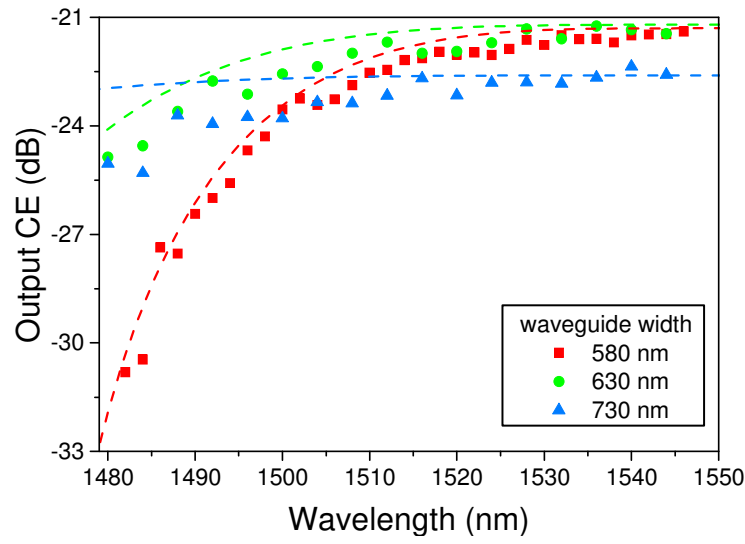

Fig. 4. Measured (symbols) and simulated (dashed lines) output conversion efficiency as a function of the signal wavelength for three different waveguide widths. The waveguide height is constant at $290 \mathrm{~nm}$.

From the output spectra, the output $\mathrm{CE}$ is measured while scanning the input signal wavelength. Throughout this work, the $\mathrm{CE}$ is defined as output $\mathrm{CE}$, i.e. the power ratio between idler and signal at the waveguide output:

$$
C E=\frac{P_{i}(L)}{P_{s}(L)},
$$

where $i$ and $s$ refer to idler and signal, respectively, and $L$ defines the waveguide length. The resulting $\mathrm{CE}$ spectra are shown in Fig. 4.

The CE spectra show a FWM half-bandwidth, measured at the 3-dB drop, in excess of $50 \mathrm{~nm}$ for all the waveguide widths investigated. However, the bandwidth increases by widening the waveguide from $580 \mathrm{~nm}$ to $730 \mathrm{~nm}$, where a halfbandwidth of more than $70 \mathrm{~nm}$ is reported, mainly limited by the tuning range of our signal laser. The numerically simulated conversion bandwidths, based on estimated dispersion (Fig. 3), are also reported in Fig. 4 and good agreement is shown between simulations and measurements. While these results would suggest that even wider waveguide may further increase the spectral window where the wavelength converter could operate, the increased dimensions lead to an increase in the effective mode area, thus lowering the mode confinement and the nonlinear effects and the dispersion becomes normal at the pumping wavelength. As a width of $630 \mathrm{~nm}$ was already sufficient to reach a half-bandwidth of approx. $60 \mathrm{~nm}$ covering the full telecommunication C-band and most of the O- and Lbands, such a waveguide cross-section of $290 \times 630 \mathrm{~nm}^{2}$ was chosen for the following analysis.

\section{Characterization of AN OPTIMIZED WaVeguide}

After choosing the cross-section, the waveguide length was considered. For this investigation, the signal-pump spacing is fixed to $10 \mathrm{~nm}$ and the pump power has been set to $18 \mathrm{dBm}$, as in the previous analysis. The results are shown in Fig. 5(a), clearly confirming that the output $\mathrm{CE}$ grows linearly with the waveguide length indicative of a non-depleted pump, low linear loss and no nonlinear loss. From the linear loss, an effective length of around $19 \mathrm{~mm}$ can be estimated. Additionally, numerical simulations show an expected optimal length as large as $31 \mathrm{~mm}$. Longer waveguides are therefore expected to lead to even higher $\mathrm{CE}$, however at the expense of a slight narrowing of the conversion bandwidth.

Notice that, in order to guarantee a fair comparison, the waveguides have been designed with a serpentine shape that kept the number of bends constant, regardless of the waveguide length. This choice prevents the impact of bending loss from affecting the comparison. Furthermore, compared to the results of Fig. 4, the sample used for producing the results of Fig. 5. a) shows higher coupling loss, possibly due to poorer cleaving of the chip, leading to lower $\mathrm{CE}$ values.

The final CE optimization parameter is the pump power. The impact of the pump power coupled in the waveguide on the achievable CE is reported in Fig. 5(b) for a 9-mm long waveguide with $1.4 \mathrm{~dB} /$ facet of coupling loss. The overall insertion loss of such a waveguide is $5 \mathrm{~dB}$. The CE shows the expected quadratic growth with pump power, without any signs of saturation due to nonlinear loss, confirming the suitability of the $\mathrm{AlGaAsOI}$ platform for nonlinear signal processing.

Finally, in Fig. 5.c), the CE bandwidth is shown for such a 9-mm long waveguide with an input pump power of $20.5 \mathrm{dBm}$. A maximum $\mathrm{CE}$ of $-12 \mathrm{~dB}$ can be achieved with a 3-dB halfbandwidth of approximately $55 \mathrm{~nm}$.

This waveguide with $20.5 \mathrm{dBm}$ of input pump power is used in the following system demonstrations.

\section{EXPERIMENTAL SETUP}

The experimental setup for the wavelength conversion of M-QAM is shown in Fig. 6. A $10 \mathrm{GBd}$ M-QAM signal is generated by modulating an external cavity laser (ECL, $100 \mathrm{kHz}$ linewidth) with an IQ modulator driven by a 64$\mathrm{GSa} / \mathrm{s}$ arbitrary waveform generator (AWG). The signal is then amplified in an EDFA followed by a 1-nm full-width half-maximum OBPF for out-of-band ASE noise suppression and injected into the wavelength converter already described in Section IV] The signal polarization is aligned to the TE mode of the 9-mm long waveguide by maximizing the CE and the pump power is set to $20.5 \mathrm{dBm}$. At the waveguide output, two OBPFs are used to select the idler, with an EDFA 

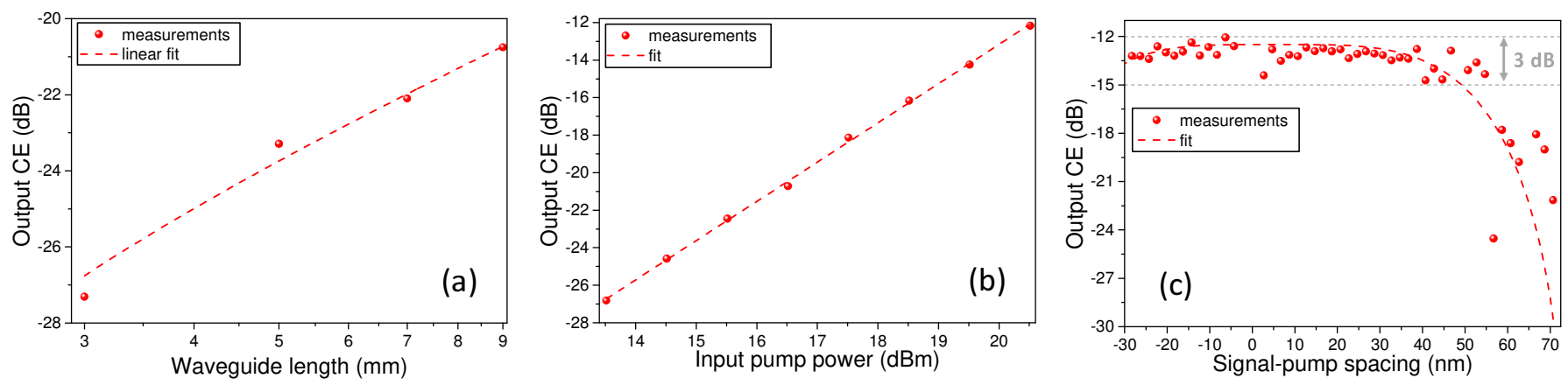

Fig. 5. $290 \times 630 \mathrm{~nm}^{2}$ waveguide: (a) output $\mathrm{CE}$ as a function of the waveguide length (measurements and linear fit with slope $=1$ ), (b) output $\mathrm{CE}$ as a function of the input pump power (measurements and linear fit with slope $=2$ ) and (c) measured (symbols) and simulated (dashed lines) output CE spectrum as a function of the signal-pump spacing for a 9-mm long waveguide and $20.5 \mathrm{dBm}$ of input pump power.

in between to compensate for their losses. An $80-\mathrm{GSa} / \mathrm{s}$ preamplified coherent receiver (33-GHz analog bandwidth) with a standard $100-\mathrm{kHz}$ local oscillator (LO) is used for reception followed by offline digital signal processing (DSP). The DSP chain included low-pass filtering, frequency offset estimation, time-domain equalization, carrier phase recovery, demapping, decoding (in the case of 256-QAM) and error counting. In order to vary the optical signal-to-noise ratio (OSNR) at the receiver side, a noise loading stage based on an ASE source is added at the receiver input.

The two modulation formats considered in the following system demonstrations are 64-QAM and 256-QAM. The forward error correction (FEC) overhead of the two modulations has been chosen such that they lead to similar net data rates, making the results more easily comparable. This choice resulted in different DSP chains for the two signals as discussed below.

In the case of 64-QAM, the uncoded data consist of two decorrelated segments of a pseudo-random bit sequence (PRBS) of length $2^{23}-1$. At the receiver side, a multimodulus algorithm (MMA) and Kalman filtering are employed for equalization and carrier phase recovery, respectively. A hard-decision (HD) FEC scheme with $7 \%$ overhead is assumed [34], leading to a net datarate of $55.8 \mathrm{Gbit} / \mathrm{s}$. In order to evaluate the performance of the wavelength converter, the pre-HD-FEC bit error ratio (BER) is used, benchmarking the generated idler quality against the back-to-back signal measured at the transmitter output. The pre-HD-FEC BER is chosen as metric as it allows to reliably predict the post-FEC BER when a HD code is used [35]. The pre-HD-FEC threshold here considered for error-free performance corresponds to a BER $=3.8 \times 10^{-3}$ and will be referred to as 'HD-FEC threshold'. The pre-FEC BER is measured counting more than 100 errors for all the OSNR levels considered, ensuring the statistical relevance of the measurements.

In the case of 256-QAM, the increased complexity of the modulation format requires more refined equalization and carrier recovery schemes, as well as soft-decision (SD) FEC with a higher overhead. At the transmitter, quadrature phaseshift keying (QPSK) pilot symbols, to be used for equalization and phase noise tracking, are interleaved with the 256-QAM random data at a rate of $10 \%$. Additionally, SD-FEC encoding is performed using a standard concatenated scheme, consisting of an inner irregular low-density parity-check (LDPC) code of length 64800 bits, and an outer Bose, Chaudhuri, and Hocquenghem $(\mathrm{BCH}) \mathrm{HD}$ code which removes the error floor [36]. At the receiver side, pilot-assisted constant modulus algorithm (CMA) equalization [37] is performed, followed by carrier phase recovery using a trellis-based method [38], iterative demapping and soft-FEC decoding (5 demapping iterations with 50 LDPC iterations in each) [36]. The equalizer taps trained on the pilots are linearly interpolated and applied to the entire received sequence [37]. The total FEC overhead is $33 \%$ leading to a net data rate of $54.6 \mathrm{Gbit} / \mathrm{s}$, after subtraction of both FEC and pilots overheads. It has been shown that the pre-SD-FEC BER is not a reliable measure to estimate system performance after decoding when SD-FEC are used. [35]. The more complex processing performed and the choice to encode the transmitted data stream have the significant advantage of not only allowing to extract pre- and post-FEC BER from the measurements but also to estimate the achievable information rate (AIR). The AIR is estimated through the mutual information (MI) defined as $I(X ; Y)=H(X)-H(X \mid Y)$, where $H(\cdot)$ is the entropy function. The entropy of the input $H(X)$ for uniformly distributed input QAM symbols (as is the case here) is found as $H(X)=1 /(1+P) \log _{2}(|\mathcal{X}|)$, where $\mathcal{X}$ is the set of constellation symbols (e.g. for 256QAM, $|\mathcal{X}|=256$ ), and the pilot rate $P$ is taken into account (in this paper, $P=0.1$ ). As the posterior probability distribution $p(x \mid y)$ is not available for the fiber channel in closed form, an auxiliary probability distribution derived from the phaserecovery algorithm is assumed for estimating the conditional entropy $H(X \mid Y)$ [38]. The AIR is here measured in bit/QAM symbol. When normalized by the occupied bandwidth, the AIR provides the spectral efficiency in bits $/ \mathrm{s} / \mathrm{Hz}$. For more details on the AIR estimation, refer to [35], [38] The postSD-FEC BER is considered error-free for values below $10^{-4}$, which are correctable by the outer $\mathrm{BCH}$ code. The AIR theoretically required to successfully decode the signal errorfree is $6 \mathrm{bit} / \mathrm{QAM}$ symbol assuming an ideal decoder (the concatenated FEC scheme considered here is near-ideal in this sense). Such threshold will be referred to as 'SD-FEC threshold' in the following. The BER and AIR values have been calculated on more than 200.000 symbols (more than $10^{6}$ 


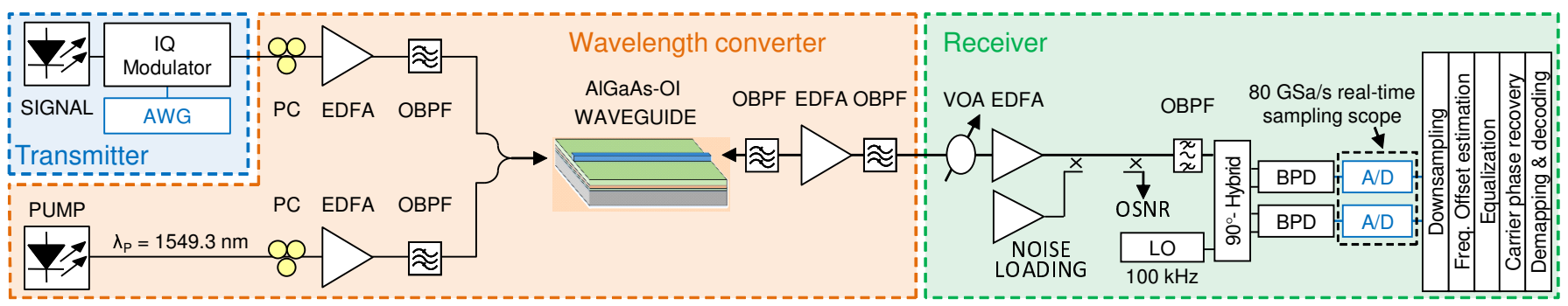

Fig. 6. Experimental setup: 256-QAM transmitter, single-pump wavelength converter based on the AlGaAsOI nano waveguide and pre-amplified converter receiver with offline digital signal processing.

information bits) in all cases, ensuring reliable measurements down to the required post-FEC BER threshold for error-free transmission (similar to the 64-QAM threshold, at least 100 errors are seen, ensuring statistically relevant BER estimation).

The necessary conditions for error-free demodulation of the two modulation formats considered in this work to test the performance of the wavelength converter are summarized in Table []

TABLE I

NECESSARY CONDITIONS FOR ERROR-FREE DEMODULATION OF THE TWO MODULATION FORMATS CONSIDERED TO BENCHMARK THE WAVELENGTH CONVERTER

\begin{tabular}{c|c|c} 
Modulation & FEC & Error-free threshold \\
\hline 64-QAM & HD-FEC, 7\% overhead & pre-FEC BER $=3.8 \times 10^{-3}$ \\
\hline 256-QAM & SD-FEC, 33\% overhead & AIR $=6$ bits/QAM symbol
\end{tabular}

Being related to either HD-FEC (64-QAM) or SD-FEC (256-QAM), in the following, the two thresholds are referred to as HD-FEC threshold and SD-FEC threshold, respectively. Two modulation formats are considered in order to demonstrate modulation-format independent operation and, more importantly, to allow testing that the wavelength converter fulfills different requirements. Given the different FEC overheads (7\% for $64-\mathrm{QAM}$ and $33 \%$ for 256-QAM), the OSNR requirements for 64-QAM are actually tighter. On the other hand, 256-QAM is less tolerant to phase-noise and nonlinear distortion introduced by the wavelength converter [39]. Additionally, the use of two signals with different DSP chains enables evaluating the performance of the converter regardless of the specific modulation format and DSP algorithms used for the analysis.

\section{OPTIMIZATION OF THE WAVELENGTH CONVERTER}

In Sections III and IV] the optimization of the wavelength converter has been focused on the nonlinear nano-waveguide alone. In this Section, instead, the focus is shifted to overall system parameters by looking at the impact of phase noise transfer from the pump to the idler and at the achievable idler OSNR. Given the stricter phase-noise and distortion tolerances, the optimization has been performed for the wavelength conversion of a 256-QAM signal. Additionally, as introduced in Section $\mathrm{V}$, the main metric used to evaluate the idler quality is AIR. Fig. 7) a) shows the AIR as a function of the received OSNR for the wavelength converted idler. The OSNR is measured over a conventional $0.1 \mathrm{~nm}$ bandwidth, the signal and pump wavelengths have been fixed to $1545 \mathrm{~nm}$ and
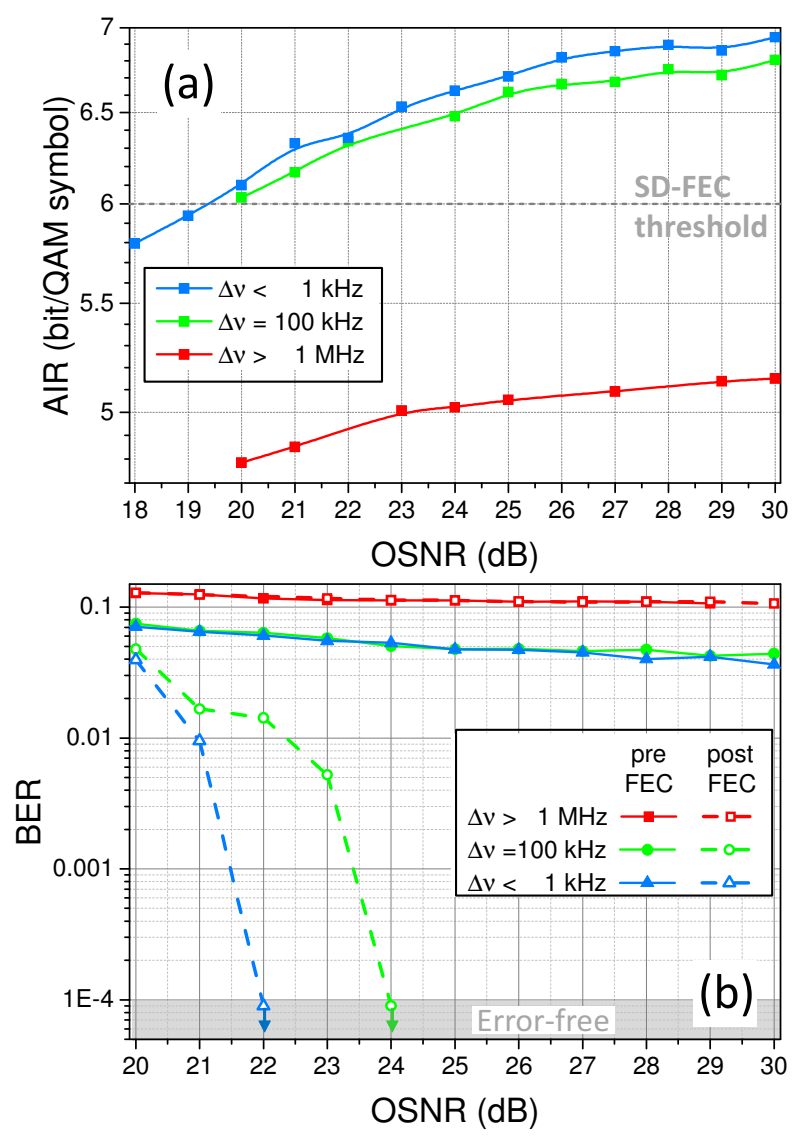

Fig. 7. Impact of pump phase noise transfer on the idler: (a) AIR and (b) preand post-SD-FEC BER as functions of the received OSNR for three different pump lasers.

$1549.3 \mathrm{~nm}$, respectively. The choice of the pump wavelength has been dictated by the need for a wavelength converter operating on the whole C-band. However, given that the dispersion is anomalous and with a low dispersion slope over the whole C-band (as highlighted by Fig. 3) a different choice of pump wavelength does not have a significant impact on the CE nor the bandwidth.

The curves correspond to pump lasers with different linewidths $\Delta \nu$, and thus different amounts of pump phase noise transferred to the idler during the FWM process. For this investigation, three pump lasers have been considered: a distributed feedback (DFB) laser with a linewidth above $1 \mathrm{MHz}$, an ECL with a linewidth of $100 \mathrm{kHz}$ and a fiber laser 


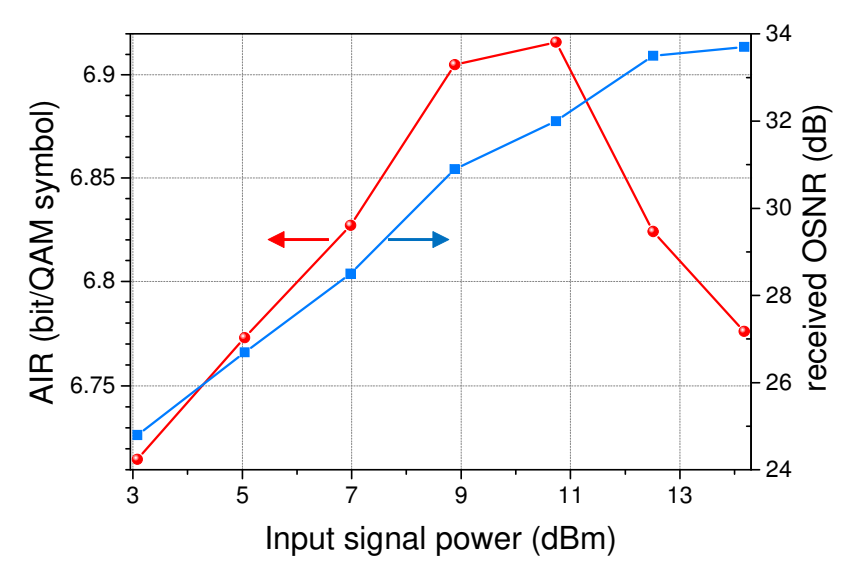

Fig. 8. Impact of input signal power: AIR (red dot) and received OSNR (blue square) as functions of the input signal power.

with sub-KHz linewidth (Koheras BasiK X-15). As can be seen in Fig. 7(a), the AIR is higher when the fiber laser is used. Using an ECL still allows to achieve the SD-FEC threshold, in contrast to a DFB pump which does not. However, using an ECL results in an additional $1 \mathrm{~dB}$ OSNR penalty compared to the narrow-linewidth fiber laser. Considering the BER results shown in Fig. 7 (b), the pre-SD-FEC BER curves for the fiber laser and ECL are almost perfectly overlapping given the high BER level achieved. As already suggested by the AIR results, however, the post-SD-FEC performance, i.e. after decoding, the superior performance of the fiber laser is clear. The OSNR penalty highlighted by the AIR of Fig. 7(a) becomes evident as $>1 \mathrm{~dB}$ additional OSNR is required for the idler to be errorfree (post-SD-FEC BER $<10^{-4}$ ) when the ECL is used as pump. The additional penalty compared to the one estimated by the AIR is due to the non-ideal FEC.

The final parameter of the wavelength converter that has been optimized is the input signal power. For a given pump power, and thus CE, the idler OSNR scales linearly with the input signal power. However, as the wavelength converter is based on a nonlinear stage, beyond a certain input signal power, the signal itself becomes affected by nonlinearity induced distortion. The trade-off between idler OSNR and nonlinear distortion is highlighted in Fig. 8. Higher OSNR results in an improved AIR only up to an input signal power of approximately $10 \mathrm{dBm}$. As the signal power is increased further, due to the strong nonlinearity in the waveguide, the signal and idler are distorted by SPM leading to an AIR degradation. This yields an optimum achieved idler OSNR of approximately $31 \mathrm{~dB}$ at the receiver side. In the following sections, the signal power is set to $9 \mathrm{dBm}$ and the fiber laser is used as pump.

\section{WAVELENGTH CONVERSION OF 64-QAM SIGNALS}

The results for wavelength conversion of 64-QAM signals are shown in Fig. 9. The pump was set to $1550 \mathrm{~nm}$ and the wavelength converter has been tested for three different pump-signal spacings: $5 \mathrm{~nm}$ (signal at $1545 \mathrm{~nm}$ ), $10 \mathrm{~nm}$ (signal at $1540 \mathrm{~nm}$ ) and $15 \mathrm{~nm}$ (signal at $1535 \mathrm{~nm}$ ). These values correspond to a maximum signal wavelength shift of
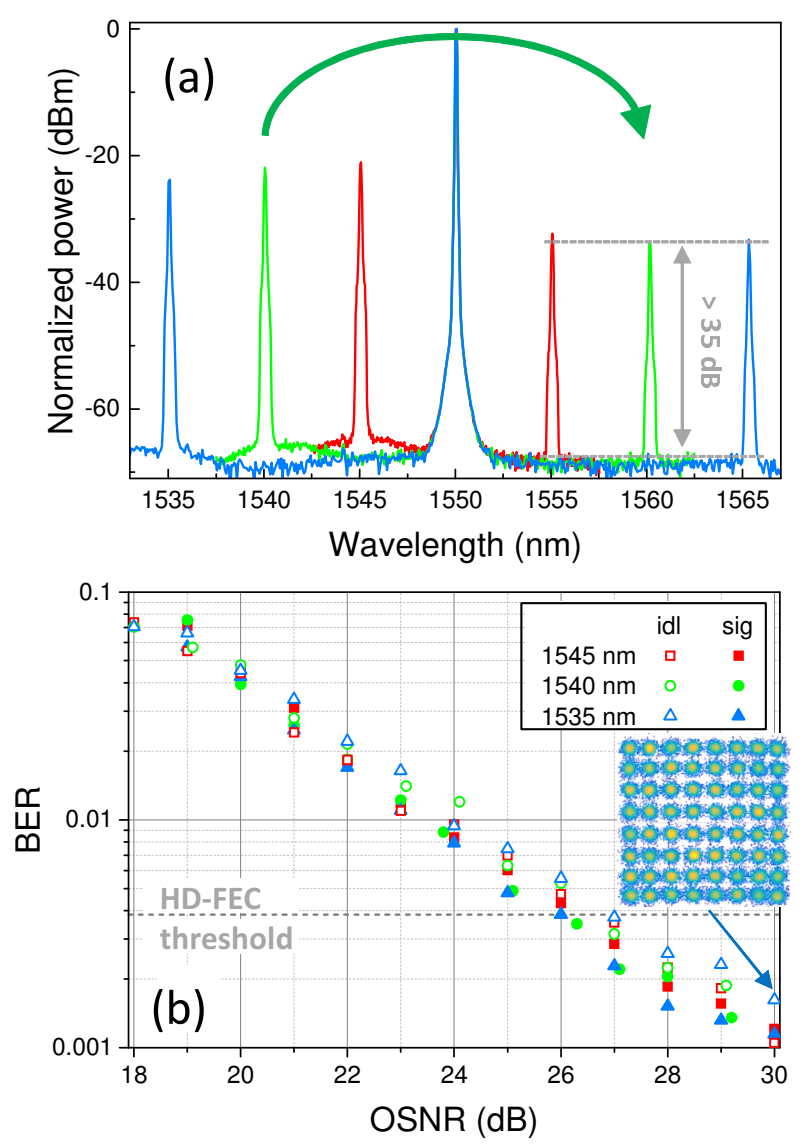

Fig. 9. 64-QAM wavelength conversion: (a) optical spectra at the waveguide output showing idler OSNR above $30 \mathrm{~dB}$ and (b) pre-HD-FEC BER performance as a function of the received OSNR. Inset in (b) shows a constellation diagram at maximum OSNR for the worst idler (signal at $1535 \mathrm{~nm}$ ).

$30 \mathrm{~nm}$, from $1535 \mathrm{~nm}$ to $1565 \mathrm{~nm}$, i.e. covering almost the full telecommunication C-band.

As can be seen in Fig. 9 a), the optical spectra at the output of the waveguide show idler OSNR levels in excess of $35 \mathrm{~dB}$. At the receiver side, the maximum OSNR is reduced to approximately $30 \mathrm{~dB}$ due the sub-optimum scheme used to suppress pump and signal waves, resulting in significant loss.

The uncoded BER is displayed as a function of the received OSNR in Fig. 9 (b) for the three signal-idler pairs. For all the signal wavelengths considered, the idler OSNR is sufficient to reach a BER below the HD-FEC threshold with more than $3 \mathrm{~dB}$ of margin. Furthermore, the OSNR penalty from the conversion, defined as the difference in required OSNR between idler and signal at a BER $=3.8 \times 10^{-3}$ (HD-FEC threshold), ranges between $0.4 \mathrm{~dB}$ for the $1545-\mathrm{nm}$ signal to $0.9 \mathrm{~dB}$ for the $1535-\mathrm{nm}$ signal.

\section{WAVELENGTH CONVERSION OF 256-QAM SIGNALS}

The results for the 256-QAM wavelength conversion (pump wavelength of $1549.3 \mathrm{~nm}$ ) are shown in Fig. 10 with the optical spectra reported in Fig. 10(a) and (b), for the short and long wavelength signals, respectively. Idler OSNR levels of more than $35 \mathrm{~dB}$ are reported for all the signal wavelengths under investigation. In this case, the higher complexity of 

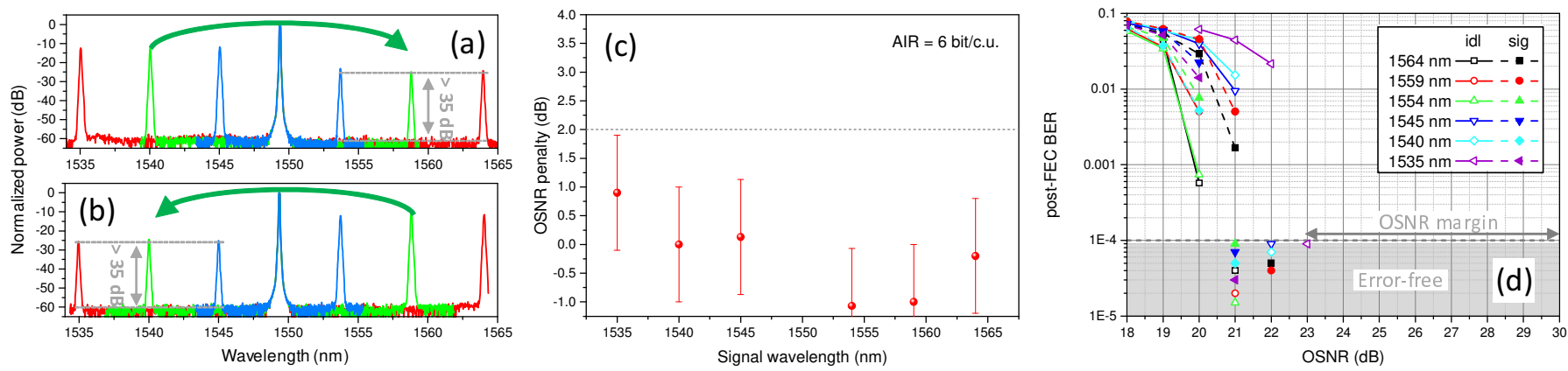

Fig. 10. 256-QAM wavelength conversion: (a) and (b) optical spectra at the waveguide output for signals on the short (a) and long (b) wavelength side of the pump showing idler OSNRs above $35 \mathrm{~dB}$; (c) OSNR penalty as a function of the signal wavelength and (d) post-FEC BER as a function of the received OSNR for all six signal-idler pairs. The symbols in the error-free area of (d) are for illustration purposes ( 0 errors measured), leading to a reliable BER $<10^{-4}$.

the modulation format considered, as well as the steepness of the BER after decoding, highlighted a slight wavelength dependence of our receiver already in a back-to-back configuration. In order to remove such a dependence and focus on the characterization of the wavelength converter, six pairs of signal-idler configurations are measured. First, the signal is placed on the short-wavelength side of the pump as for the results of Section VII and the same three signal wavelengths have been considered (spectra of Fig. 10(a)). Then, the signal has been placed on the long-wavelength side of the pump, at the wavelengths where the idlers are generated in the previous scenario (spectra of Fig. 10(b)). This approach allows to measure the transceiver OSNR penalty caused by sweeping a 29-nm wavelength range for the signal in back-to-back and use such numbers to extract the penalty due to the wavelength conversion from the overall difference in the required OSNR between signal and idler.

From the AIR versus OSNR curves, the OSNR penalty has been extracted focusing on the AIR $=6 \mathrm{bit} / \mathrm{QAM}$ symbol threshold (SD-FEC threshold), and splitting the contribution from the receiver side (estimated as described above) of the contribution from the wavelength converter itself. The wavelength converter penalty is reported in Fig. 10 (c) as a function of the signal wavelength. Error bars are included to account for the finite length of the transmitted sequences and uncertainties in the OSNR measurements. Even accounting for the error bars, an OSNR penalty below $2.0 \mathrm{~dB}$ can be achieved over the whole 29-nm wavelength window. Remark that the OSNR requirements are lower than for 64-QAM (see Fig. 9) due to the more powerful FEC employed for 256-QAM.

Finally, Fig. 10(d) reports the decoded BER curves for the six signal-idler pairs. After SD-FEC decoding, all the idlers could be measured error-free (over more than $10^{6}$ bits) at a maximum required OSNR of $23 \mathrm{~dB}$. Such a requirement is well below the idler OSNR $>30 \mathrm{~dB}$ provided at the wavelength converter output, as shown in Fig. 10(a),(b). The idler OSNR at the converter output is therefore expected to allow for subsequent transmission of the wavelength converted signals.

\section{CONCLUSION}

An all-optical wavelength converter based on a 9-mm long AlGaAs-On-Insulator nano-waveguide was experimen- tally demonstrated for 10-GBd 64-QAM and 256-QAM signals. The wavelength converter has been first characterized by optimizing the nonlinear waveguide in terms of waveguide cross-section, length and pump power by maximizing the achievable conversion efficiency and conversion bandwidth. Then, the wavelength converter has been studied from a system perspective by focusing on the pump-laser induced phase noise on the idler and the trade-off between OSNR degradation and SPM induced nonlinear distortion. With such optimized converter,the OSNR penalty after conversion has been studied by using 64-QAM and 256-QAM, at approx. the same net data rate but with different OSNR and distortion requirements. For the 64-QAM signal, less than $1 \mathrm{~dB}$ penalty is reported compared to the back-to-back signal at the HDFEC threshold (BER $=3.8 \times 10^{-3}, 7 \%$ overhead). The system investigation has been then extended to 256-QAM, by using achievable information rate (AIR) as a figure of merit to define the conversion penalty under SD-FEC operation. Low OSNR penalty $(<2.0 \mathrm{~dB})$, at the target AIR of $6 \mathrm{bit} / \mathrm{QAM}$ symbol (33\% SD-FEC threshold), has been shown spanning a 29-nm bandwidth with received idler OSNR $>30 \mathrm{~dB}$ (OSNR margin $>7 \mathrm{~dB}$ ) to potentially enable further transmission.

\section{ACKNOWLEDGMENT}

This work was supported by the DNRF centre of excellence, SPOC, ref. DNRF123, the DFF-4005-00558 project NANOSPEC, the SIMOF project, and the Villum Fonden center of excellence NATEC. NKT Photonics A/S is acknowledged for kindly providing the narrow linewidth fiber laser.

\section{REFERENCES}

[1] X. Wang et al., "Efficient all-optical wavelength converter placement and wavelength assignment in optical networks," in Proc. of Optical Fiber Communication Conference (OFC), 2016, paper W2A52.

[2] Z. Tong et al., "Towards ultrasensitive optical links enabled by low-noise phase-sensitive amplifiers," Nature Photonics, Vol. 5, p. 430 (2011).

[3] R. Slavik et al., "All-optical phase and amplitude regenerator for nextgeneration telecommunications systems," Nature Photonics, Vol. 4, p. 690 (2011).

[4] F. Da Ros et al., "Phase regeneration of DPSK signals in a silicon waveguide with reverse-biased p-i-n junction," Optics Express, Vol. 22, no. 5 , p. 5029 (2014).

[5] M.A. Ettabib et al., "All-optical phase regeneration with record PSA extinction ratio in a low-birefringence silicon germanium waveguide ," IEEE Journal of Lightwave Technology, vol. 34, no. 17, p. 3993 (2016). 
[6] D. M. Pepper and A. Yariv, "Compensation for phase distortions in nonlinear media by phase conjugation," Optics Letters, Vol. 5, no. 2, p. 59 (1980).

[7] A.D. Ellis et al., " $4 \mathrm{~Tb} / \mathrm{s}$ transmission reach enhancement using $10 \times 400$ $\mathrm{Gb} / \mathrm{s}$ super-channels and polarization insensitive dual band optical phase conjugation," IEEE Journal of Lightwave Technology, vol. 34, no. 8, p. 1717 (2016).

[8] I. Sackey et al., "Kerr nonlinearity mitigation in $5 \times 28$-GBd PDM 16QAM signal transmission over a dispersion-uncompensated link with backward-pumped distributed Raman amplification," Optics Express, Vol. 22, no. 22, p. 27381 (2014).

[9] D. Vukovic et al., "Multichannel nonlinear distortion compensation using optical phase conjugation in a silicon nanowire," Optics Express, Vol. 23, no. 3, p. 3640 (2015).

[10] A. H. Gnauck, et al., "All-optical tunable wavelength shifting of a 128Gbit/s 64-QAM signal," in European Conference on Optical Communication (ECOC), 2012, paper Th2F2.

[11] H. Nguyen Tan et al., "On the cascadability of all-optical wavelength converter for high-order QAM formats," IEEE Journal of Lightwave Technology, vol. 34, no. 13, p. 3194 (2016).

[12] M.D. Pelusi et al., "Wavelength conversion of high-speed phase and intensity modulated signals using a highly nonlinear chalcogenide glass chip," IEEE Photonics Technology Letters, Vol. 22, no. 1, p. 3 (2010).

[13] H. Hu et al., "Tunable all-optical wavelength conversion based on cascaded SHG/DFG in a Ti:PPLN waveguide using a single CW control laser," IEEE Photonics Journal, Vol. 4, no. 5, p. 1396 (2012).

[14] T. Richter et al., "PPLN-waveguide based tunable wavelength conversion of QAM data within the C-band," IEEE Photonics Technology Letters, Vol. 25, no. 21, p. 2085 (2013).

[15] C. Lacava et al., "Nonlinear silicon photonic signal processing devices for future optical networks," Applied Science, Vol. 7, no. 1, p. 103 (2017).

[16] M.A. Ettabib et al., "FWM-based wavelength conversion of 40 Gbaud PSK signals in a silicon germanium waveguide," Optics Express, Vol. 21, no. 14 , p. 16683 (2013).

[17] C.J. Krückel et al., "Continuous wave-pumped wavelength conversion in low-loss silicon nitride waveguides," Optics Letters, Vol. 40, no. 6, p. 875 (2015).

[18] F. Da Ros et al., "Wavelength conversion of QAM signals in a low loss CMOS compatible spiral waveguide," in APL Photonics Vol. 2, no. 4, p. 046105 (2017)

[19] K. Hammani et al., "Optical properties of silicon germanium waveguides at telecommunication wavelengths," Optics Express, Vol. 21, no. 14, p. 16690 (2013).

[20] M.A. Ettabib et al., "Wavelength conversion of complex modulation formats in a compact SiGe waveguide," Optics Express, Vol. 25, no. 4, p. 3252 (2017).

[21] J.S. Aitchison et al., "The nonlinear optical properties of AlGaAs at the half band gap," IEEE Journal of Quantum Electronics, Vol. 33, no. 3, p. 341 (1997).

[22] K. Dolgaleva et al., "Compact highly-nonlinear AlGaAs waveguides for efficient wavelength conversion, Optics Express, Vol. 19, no. 13, p. 12440 (2011).

[23] C. Lacava et al., "Nonlinear properties of AlGaAs waveguides in continuous wave operation regime, Optics Express, Vol. 22, no. 5, p. 5291 (2014).

[24] J.J. Wathen et al., "Efficient continuous-wave four-wave mixing in bandgap-engineered AlGaAs waveguides," Optics Letters, Vol. 39, no. 11 , p. 3161 (2014).

[25] M. Pu et al., "AlGaAs-on-insulator nanowire with $750 \mathrm{~nm}$ FWM bandwidth, $-9 \mathrm{~dB}$ CW conversion efficiency, and ultrafast operation enabling record Tbaud wavelength conversion," in in Proc. of Optical Fiber Communication Conference (OFC), 2015, paper Th5A3.

[26] L. Ottaviano et al., "Low-loss high-confinement waveguides and microring resonators in AlGaAs-on-insulator," Optics Letters, Vol. 41, no. 17, p. 3996 (2016)

[27] F. Da Ros et al., "Broadband and efficient dual-pump four-wave-mixing in AlGaAs-on-insulator nano-waveguides," in Proc. of Conference on Lasers and Electro-Optics (CLEO), 2016, paper SM1E.3.

[28] F. Da Ros et al., "Characterization of a wavelength converter for 256-QAM signals based on an AlGaAs-On-Insulator nano-waveguide," in European Conference on Optical Communication, (ECOC), 2016, paper W.3.C.3.

[29] F. Da Ros et al., "Phase-sensitive four-wave mixing in AlGaAs-OnInsulator nano-waveguides," in Proc. IEEE Photonics Conference (IPC), 2016, paper WB1.1.
[30] H. Hu et al., "Single-source AlGaAs frequency comb transmitter for 661 Tbit/s data transmission in a 30-core fiber" in Proc. of Conference on Lasers and Electro-Optics (CLEO), 2016, paper JTh4C.1.

[31] M. Pu et al., "Efficient frequency comb generation in AlGaAs-oninsulator," Optica, Vol. 3, no. 8, p. 823 (2016).

[32] M. Pu et al., "Ultra-low-loss inverted taper coupler for silicon-oninsulator ridge waveguide," Optical Communications, Vol. 283, no. 19, p. 3678 (2010).

[33] M.A. Afromowitz, "Refractive index of $\mathrm{Ga}_{1-1} \mathrm{Al}_{x} \mathrm{As}$," Solid State Communications, Vol. 15, no. 1, p. 59 (1974).

[34] ITU-T Rec. G.975.1 (02/2004) Forward error correction for high bit-rate DWDM submarine systems.

[35] A. Alvarado et al., "Replacing the soft-decision FEC limit paradigm in the design of optical communication systems," IEEE Journal of Lightwave Technology, vol. 34, no. 2, p. 707 (2016).

[36] Digital Video Broadcasting Second generation (DVB S2), ETSI EN 302 307, V1.2.1, April 2009.

[37] M.P. Yankov et al., "Experimental analysis of pilot-based equalization for probabilistically shaped WDM systems with 256QAM/1024QAM," in Proc. of Optical Fiber Communication Conference (OFC), 2017, paper W2A.48.

[38] L. Barletta et al., "Pilot-aided trellis-based demodulation," IEEE Photonics Technology Letters, Vol. 25, no. 13, p. 1234 (2013).

[39] M.P. Yankov et al., "Low-complexity tracking of laser and nonlinear phase noise in WDM optical fiber systems, IEEE Journal of Lightwave Technology, vol. 33, no. 23, p. 4975 (2015). 